\title{
Los aportes epistemológicos del pensamiento antropológico sobre el Estado moderno y las etnografias sobre sus prácticas cotidianas ${ }^{1}$
}

\author{
Juan Felipe Garcia Arboleda ${ }^{2}$ \\ Pontificia Universidad Javeriana, Bogotá, Colombia ${ }^{3}$ \\ jgarciaa@javeriana.edu.co \\ Recibido: 21 de julio 2015 \\ Aceptado: 30 de octubre de 2015 \\ Disponible en línea: 9 de mayo de 2016
}

\footnotetext{
Artículo de revisión realizado para la construcción del marco teórico del proyecto de tesis doctoral titulado "Justicia Transicional en la Isla de Papayal: etnografias sobre el Estado en un contexto de conflicto por el acceso, uso y control de los recursos naturales", adscrito al Doctorado en Antropologia de la Universidad de los Andes.

2 Abogado, Magíster en Urbanismo de la Universidad Nacional de Colombia. Es candidato al Doctorado en Antropología de la Universidad de Los Andes. Su último libro se titula El lugar de las victimas en Colombia.

3 Profesor asistente de la Facultad de Ciencias Juridicas de la Pontificia Universidad Javeriana en Bogotá. Miembro del Grupo de Investigación en Justicia Social
} 


\title{
Los aportes epistemológicos del pensamiento antropológico sobre el Estado moderno y las etnografias sobre sus prácticas cotidianas
}

\section{Resumen}

Este trabajo es un artículo de revisión que pretende reconstruir episodios significativos para la disciplina antropológica en su experiencia de observación -a través del método etnográfico- de eso que llamamos 'el poder'. Argumento que dicha experiencia derivó en un hallazgo epistemológico central para el conjunto de las ciencias sociales al que denomino la desontologización del Estado. Exploro tres ejemplos de diversificación de los objetos de observación etnográfica que prepararon el camino para dicha desontologización. Declino la pretensión de escrutinio crítico y exhaustivo de las etnografias sobre el Estado y al final invito a la reflexión sobre tres de los problemas de orden teórico conceptual que han surgido a partir de los resultados de investigación de dichas etnografias: el análisis de los mitos y los ritos del Estado, el problema del fundamento de su soberanía y la pregunta por el significado de sus márgenes.

Palabras clave: antropología política; etnografias sobre el Estado; soberanía estatal; mitos de Estado; ritos de Estado; márgenes del Estado

\section{Epistemological Contributions of Anthropological Thought on the Modern State and the Ethnographies on its Daily Practices}

\begin{abstract}
This work is a review article aiming to rebuild significant episodes for the anthropology discipline in its observation experience -through the ethnographical method- of what we call 'power'. I argue that said experience led to an epistemological central finding for the whole group of social sciences which I coined as de-ontology of the State. I explore three examples of diversification of the ethnographic observation objects which paved the road for said de-ontology. I deny any pretension of performing a critical and comprehensive examination of the ethnographies on the State, and finally, I call to reflection on three of the conceptual-theoretical problems which have appeared from the results of the research on said ethnographies: the analysis of the myths and rituals of the State; the problem of the foundation of sovereignty; and the question around the meaning of its margins.

Keywords: political anthropology; ethnographies on the State; state sovereignty; myths of the State; rituals of the State; margins of the State
\end{abstract}

\section{Aportes epistemológicos do pensamento antropológico sobre o Estado moderno e as etnografias das suas práticas cotidianas}

\section{Resumo}

Este trabalho é um artigo de revisão que visa reconstruir episódios significativos para a disciplina antropológica na sua experiência de observação -através do método etnográfico- de aquilo que chamamos de 'poder'. Argumento que tal experiência derivou em uma descoberta epistemológica central para o conjunto das ciências sociais que nomeio desontologização do Estado. Exploro três exemplos de diversificação dos objetos de observação etnográfica que prepararam o caminho para tal desontologização. Rejeito a pretensão de escrutínio crítico e exaustivo das etnografias sobre o Estado e por fim convido à reflexão sobre três dos problemas de ordem teórica conceitual que surgiram a partir dos resultados de pesquisa de essas etnografias: a análise dos mitos e ritos do Estado, o problema do fundamento da sua soberania e a pergunta pelo significado das suas margens.

Palavras-chave: antropologia politica; etnografias sobre o Estado; soberania estatal; mitos de Estado; ritos de Estado; margens do Estado 
En la ciencia jurídica el Estado moderno ${ }^{4}$ es un supuesto epistemológico que reduce su objeto de estudio a las normas que son efecto de aquel ${ }^{5}$. Tal como lo ha señalado recientemente Felipe Cárdenas, la situación es análoga en la ciencia política, pues enfoques de esta disciplina pretenden reducir 'lo politico' a una forma particular de 'lo politico' ligado a la experiencia del Estado moderno (Cárdenas-Támara, 2014).

La sociología y la antropología, de otro lado, con sus particulares objetos de estudio, abdicaron por un buen período de tiempo de considerar el Estado moderno como un fenómeno directo de observación. O bien lo expulsaban de su horizonte de investigación o incluso ponian en duda su existencia empírica.

Pienso que se llega a esta situación cuando a un ente se le sacraliza a tal nivel que ni siquiera se le pueden formular las preguntas ontológicas fundamentales: ¿eres?, ¿qué eres?, ¿cómo has llegado a ser?, ¿siempre has sido?, ¿siempre serás?, ¿eres único? En ese sentido considero que es relevante aunar esfuerzos desde las ciencias sociales para construir una ontologia del Estado y al hacerlo, de alguna manera considerar su desontologización. En la presente revisión quisiera presentar hallazgos significativos que la antropologia ha derivado de la aplicación de su método etnográfico, los cuales resultan ser aportes epistemológicos centrales en dicho proceso de desontologización.

4 En el presente trabajo se entiende Estado moderno en la línea desarrollada por Pierre Bourdieu para quien se trata de un artefacto social que tiene la capacidad de conferir a un arbitrio cultural europeo (conjunto particular de normas-dogmas-valores) todas las apariencias de naturalidad y generalidad, lo que denomina capital simbólico. De acuerdo al sociólogo francés, la especial acumulación de capital simbólico por parte del Estado debe entenderse como producto de un largo proceso histórico que se gesta desde el siglo XVII en el que se acumulan otros capitales como el militar, el económico, el informacional y el jurídico, lo que provee al Estado la capacidad de enunciar juicios de verdad (Bourdieu, 1997, p. 57)

$5 \mathrm{El}$ argumento canónico de H. L. A Hart es medianamente simple. En su trabajo que tiene como pretensión delimitar el objeto de estudio del derecho, establece que en toda sociedad moderna las personas reconocen la existencia o no de un Estado constitucional. Es decir la existencia de un sistema de producción y aplicación de normas en donde existe una norma secular suprema que garantiza derechos subjetivos, que prescribe los debidos procesos para producirlas y aplicarlas y que establece las entidades competentes para hacerlo (Hart, 1998). 


\title{
La paradójica relación de la antropología política con el Estado moderno
}

\begin{abstract}
En 1940 se publicó el texto editado por Meyer Fortes y E.E EvansPritchard titulado African Political Systems. En este libro se juntaron los trabajos etnográficos de una generación de antropólogos que derivaban su conocimiento de dos experiencias particulares. Por un lado la tradición teórica del estructuralismo-funcionalismo, que en aras de analizar el sistema político de las sociedades primitivas delimitaba el objeto de estudio a las instituciones, normas, valores e ideales que de manera reiterada son obedecidas por los miembros de estas sociedades. Por otro lado la experiencia inglesa de colonización de África que implicó que las investigaciones se realizaran en territorios africanos colonizados por Inglaterra en un contexto en el que el gobierno colonial tenía un particular interés por conocer las formas politicas de los nativos para profundizar sus estrategias de gobierno (Lewellen, 2003, p. 7).
\end{abstract}

Estas experiencias que enmarcan los trabajos contenidos en el libro de Fortes y Evans-Pritchard ayudan a comprender las tipologias centrales que dichos estudios comparten como punto de partida de sus análisis: las poblaciones en las que desarrollan su etnografia se clasifican en aquellas que tienen un Estado primitivo y aquellas sociedades sin Estado. Las primeras son las que han centralizado sus autoridades políticas y judiciales, y las últimas, las que aún no han consolidado dicha centralización (Lewellen, 2003, p. 7; Kurtz, 2001, pp. 73-74).

Estas tipologías están activas en el trabajo de Evans-Pritchard sobre los Nuer, una población de 200.000 personas en el nororiente de África, y que, de acuerdo al antropólogo inglés, podían lograr el equilibrio social a pesar de no tener un gobierno centralizado. Afirma Evans-Pritchard (2002):

The lack of governmental organs among the Nuer, $[\ldots]$ and generally, of organized political life is remarkable. Their state is an acephalous kinship state and it is only by a study of the kinship system that it can be well understood how order is maintained and social relations over wider areas are established and kept up. The 
ordered anarchy in which they live accords well their character, for it is impossible to live among Nuer and conceive of rulers ruling over them. (p. 38)

En el pasaje citado se puede apreciar que Evans-Pritchard reconoce que existen nociones nativas de autoridad y justicia, por más exóticas que ellas aparezcan a los ojos del colonizador. Asimismo subraya las tensiones que existen entre estas nociones y las europeas, anticipando las dificultades del sometimiento de estas poblaciones al gobierno colonial (Gledhill, 2000, p. 4). Aquí el trabajo de la antropología politica consiste en evidenciar que a pesar de la diferencia cultural, los nativos tienen sistemas politicos particulares que cumplen la misma función que la de los sistemas politicos occidentales.

Incluso en un contexto de validación positivista del conocimiento, el método etnográfico es postulado como una herramienta más adecuada para la investigación del poder pues permite un acceso empírico a los datos que arroja la realidad. En una audaz afirmación RadcliffeBrown (1940) establece en el prólogo de African Political Systems:

There is no such thing as the power of the state; there are only, in reality, powers of individuals -kings, prime ministers, magistrates, policemen, party bosses and voters. (p. xxiii citado en Kurtz 2001, pp.175-176)

La invitación de la antropología politica a la reflexión metodológica sobre el análisis del poder derivó en una revisión del paradigma estructuralista-funcionalista. En 1954 Edmund Leach presentó su trabajo Political Systems of Higland Burma y en 1957 Victor Turner publicó Schism and Continuity in an African Society. Aunque el tipo de relaciones sociales descritas en estos análisis difieren el uno del otro -el primero se enfocaba en la relación entre las poblaciones étnicas y el Estado nacional mientras el segundo en las relaciones entre unos cuantos individuos en un pequeña aldea-, ambos coinciden en dejar atrás la idea según la cual el objeto de estudio de un sistema político son las formas de obediencia que aparecen en la cotidianidad de la observación, las cuales, de acuerdo al paradigma vigente, preservan el 
orden y el equilibrio social. Para ellos las etnografias deben dar cuenta más bien de las tensiones y conflictos que surgen en los procesos sociales en un contexto en que la obediencia al poder se encuentra en permanente disputa (Lewellen, 2003, pp. 84-85).

Una década después, en 1964, Marc J. Swartz, Victor Turner y Arthur Tuden propusieron que la conferencia anual de la Asociación Americana de Antropología se dedicara a realizar una revisión sobre las aproximaciones teóricas en el campo de la antropología política. En 1966 editaron un libro que recopilaba los trabajos de los participantes en la conferencia. Alli los antropólogos señalaron:

Many of the papers we received centered their discussions on dynamic phenomena and processes. They considered both repetitive and radical political change, the processes of decision-making and conflict resolution, and the agitation and settlement of political issues in a variety of cultural contexts. The papers were pervaded by a "becoming" rather than a "being" vocabulary: they were full of such terms as "conflict", "faction", "struggle", "conflict resolution", "arena", "development", "process" and so forth. (2002, p. 102)

Para Swartz, Turner y Tuden, si bien la antropología tomaba prestado este vocabulario de la filosofia de Hegel (dialéctica), Marx (contradicción - lucha) y Simmel (conflicto), fue el trabajo de Max Gluckman -y su desarrollo en la llamada Escuela de Manchester- el que dio claridad al uso de estas categorías en los trabajos etnográficos. Es por ello que citan directamente las palabras de Gluckman (1965):

If we view these relations through a longish period of time, we see how various parties and supporters operate and manipulate mystical beliefs of various kinds to serve their interests. The beliefs are seen in dynamic process within day-to-day social life, and the creation and burgeoning of new groups and relationships (p. 235 citado en Swartz, Turner y Tuden, 2002, p. 103)

Lo que está en juego en esta revisión del paradigma estructuralista-funcionalista es una variación en la comprensión del fenómeno 
politico. Este pasa a ser entendido como un proceso que acontece en el día a día de la vida social y por ello mismo, precisa ser explicado como una interrelación de múltiples actores durante un período de tiempo determinado en el que lo que se constata no es la estructura de los límites del poder sino la disputa por dichos límites. En efecto, al analizar la cotidianidad de la administración del poder lo que se observa es que las personas que están involucradas en los procesos políticos -incluso aquellos que se encuentran bajo estructuras de dominación- se autoperciben en un rol protagónico, pues consideran que participan con sus acciones estratégicas en la redefinición de los límites del poder ${ }^{6}$ (Lewellen, 2003, p. 85).

Esta variación del paradigma estructural-funcionalista produce un efecto epistemológico al que denomino desontologización del poder, pues la pregunta ontológica implica la cuestión sobre el ser universal y el ser particular: el efecto que se deriva de las etnografias consiste en la sospecha radical de la existencia de una forma universal, regular y constante de limitar y estabilizar el poder. A través de esta nueva interpretación de las etnografias del ejercicio del poder en África, la antropología política demostraba la particularidad, la inestabilidad y la tensión de la estructuración del poder en tiempo real. De cierta manera se realizaba una crítica a la ontología del poder pregonada por el liberalismo desde finales del siglo XVIII, que afirmaba precisamente, la necesidad de universalizar las formas de limitar el poder diseñadas por los europeos.

Veena Das y Deborah Poole (2004) señalan que el trabajo de la antropología política referida hasta aquí comprendía el mundo de lo primitivo como un lugar nostálgico para el hallazgo del Estado, incluso cuando este no se encontraba en las sociedades que analizaban ${ }^{7}$.

6 Es pertinente advertir que los aportes epistemológicos para una desontologización del poder y del Estado no provienen exclusivamente de la antropología política. Para mencionar solo un ejemplo, el pensador político italiano Antonio Gramsci sostuvo sistemáticamente que en el Estado moderno el poder está aparentemente centralizado en las instituciones formales que lo constituyen, pero en realidad el poder descansa en las prácticas sociales cotidianas que reafirman la verdadera relación entre gobernantes y gobernados. Argumenta Gledhill que Gramsci define hegemonía "as a dynamic process of "establishment of unstable equilibria" which is shaped in significant ways by the actions and reactions of the subaltern classes" (Gledhill, 2000, 77).

7 Aquí es paradigmático el debate entre Evans-Pritchard y Pierre Clastres. Este último intenta demostrar que Evans-Pritchard no comprende el hallazgo fundamental de la antropología política 
Dicha comprensión, sostienen las antropólogas citadas, proviene del uso de la forma-Estado como un operador cultural universal (Das y Poole, 2004, p. 5). Usar la forma-Estado como operador cultural universal solo es posible si sobre dicha forma-Estado ha recaído una operación de ontologización, esto es una operación que sostiene de manera simultánea, que la forma-Estado es una norma-un postulado particular de orden- y lo normal-la forma universal de orden- como lo argumentó Jeremy Bentham para justificar el gobierno de las colonias inglesas (Chatterjee, 2011, pp. 4-5).

Tal y como lo entiendo este hallazgo central de la antropología política es el que usa Felipe Cárdenas en el ámbito de la ciencia política colombiana para realizar una crítica a ciertos enfoques que intentan circunscribir su objeto de estudio a una concepción distributiva de la ciencia política positiva que dialoga con la economía y la psicología pero que en todo caso, no son más que una experiencia de orden particular y añadiría yo, ligada a los procesos concretos de construcción del Estado moderno. De alli su llamado a reconstituir un objeto de estudio más heurístico para la ciencia política que indague por diversas experiencias de orden que permitan ampliar nuestra comprensión del fenómeno político, lo que aquí he llamado desontologizar el poder (Cárdenas-Támara, 2014).

Es la ausencia de un proyecto ontológico sobre el Estado moderno lo que bloquea en muchos espacios la reconstitución de los objetos de estudio de las ciencias sociales. Es importante subrayar que a pesar de lograr este hallazgo epistemológico, la antropología política fue incapaz, en su momento, de confrontar directamente un mundo estructurado por la expansión del colonialismo occidental y hacer la crítica a su herramienta más poderosa: el Estado moderno (Gledhill, 2000, p. 3). Esta incapacidad, argumenta Gledhill, se puede explicar por dos razones.

al evidenciar que existen sociedades sin Estado: la ausencia de este implica la eliminación de la división social entre dominadores y dominados. Afirma Pierre Clastres que "Retendremos una propiedad común que hace oponerse en bloque a las sociedades con Estado y las sociedades primitivas. Las primeras presentan, todas ellas, esa dimensión de división desconocida entre las otras. Todas las sociedades con Estado están divididas en dominadores y dominados, mientras que las sociedades sin Estado ignoran esta división" (Clastres, 2001, p. 112). Con todo, se corrobora lo que afirman Veena y Das: sin tener presencia en estas sociedades -independientemente del debate sobre los efectos de dicha ausencia- el Estado está siempre presente como categoría espectral de análisis. 
Por un lado, una huella de etnocentrismo, la cual se manifiesta en el uso acrítico del concepto de Estado de Max Weber, de acuerdo al cual el monopolio de la fuerza que realiza una autoridad centralizada es un proceso 'más racional' que otras formas de administración del poder, lo que explicaría su poder de universalización (Weber, 2006). Por otro lado esta racionalización del Estado como forma general de administración del poder de las sociedades de las que provenían los antropólogos dificultaba la posibilidad de estudiarlo como un fenómeno particular, valga decir, cultural, características que solo lograban ver en las comunidades exóticas, los sujetos específicos de estudio en la antropología como disciplina (Gledhill, 2000, pp. 10-15).

De alli la paradójica relación entre la antropología política y el Estado moderno: abandona a este último como objeto directo de estudio, pero a la vez lo postula como limite constitutivo de dicho objeto. Este limite constitutivo se presenta en un doble sentido. En primer lugar, el poder que pretende estudiar la antropología política es aquel que acontece en el margen del Estado moderno, aquel que se identifica visiblemente como 'otro'. En segundo lugar, la mirada con la que va a observar, tipificar y clasificar a ese 'otro' busca la 'presencia del Estado', su 'ausencia' o su 'relación con'. Como se dijo anteriormente, el Estado funciona como operador cultural universal o término general/normal de comparación.

\section{El giro de la antropología política (la desontologización del poder) y la diversificación de sus objetos de estudio}

Ahora bien los hallazgos sobre la inestabilidad de las formas politicas en los espacios coloniales paulatinamente derivó en la exploración de nuevos campos de observación, sobretodo aquellos en los que aparecían formas intolerables de dominación después de la Segunda Guerra Mundial en las sociedades que se autodenominan modernas (Gledhill, 2000 , p. 21). Sin pretensión alguna de exhaustividad, a continuación expongo tres caminos emprendidos por antropólogos que tienen como 
punto de partida la desontologización del poder y que, al dirigir su mirada a nuevos campos de observación de la antropología política, prepararon la ruta de la desontologización del Estado. Estos nuevos campo de observación son: los procesos de descolonización del siglo $\mathrm{XX}$, los efectos del poder en las sociedades modernas 'más allá' del Estado y la emergencia de un 'orden global'.

\section{Los procesos de descolonización del siglo $\mathrm{XX}$}

En 1973 el antropólogo árabe Talal Asad lanzó una compilación de estudios titulado Anthropology and the colonial encounter. En esta, Asad argüía que la antropología estaba arraigada en el encuentro colonial, el cual no solo había profundizado unas condiciones de asimetría política -funcional a la emergencia de la antropología como disciplina-, sino que además habia borrado el tiempo en que Oriente fue más poderoso que Occidente, velación en la que de manera cómplice participó la antropología (Vincent, 2002, pp. 129-130).

En un ensayo posterior Asad vuelve sobre el papel de la disciplina antropológica en el encuentro colonial afirmando que si bien la antropología no fue tan importante para el proyecto colonial -el cual tenía una dinámica autónoma-, el proyecto colonial fue fundamental para la antropología, la cual no contaba con dicha autonomía. Por un lado la asimetría que producía el gobierno colonial era la condición de posibilidad para proveer poblaciones y territorios para 'ser observados'. Por otro lado eran los conceptos político-jurídicos que legitimaban la colonización en el ordenamiento legal internacional los que condicionaban la producción teórica de la antropología: nociones como Estado-nación-derecho (modernos) eran opuestos a tribu-pueblo-religión (primitivos), lo que en el marco jurídico de la época justificaba la colonización (Asad, 2002, p. 134) ${ }^{8}$.

Según Asad, es esta oposición entre moderno/primitivo -fundamental en la epistemología antropológica de la primera mitad del siglo veinte- lo que hace de la secularidad moderna una religión intolerante

\footnotetext{
8 Para seguir los efectos jurídicos de estas clasificaciones revisar Alexandrowicz (1971).
} 
(una ontologización en el sentido empleado arriba), desde la cual se censuran los procesos políticos religiosos de liberación árabe y descolonización durante el siglo XX (Asad, 2002, p. 136). En ese contexto Asad aboga por una antropología crítica del poder imperial occidental que se preocupe por la alteración y emergencia de nuevos sujetos, lenguajes, poderes, grupos sociales, deseos, miedos y subjetividades derivadas del encuentro colonial (Asad, 2002, p. 139).

En 1984 otro trabajo abordó, desde una perspectiva crítica, el encuentro colonial que seguía vivo en la primera mitad del siglo XX de manera simultánea con los procesos de descolonización. En este trabajo Michael Taussig (2002, pp. 26-27) afirmaba que,

Sean cuales fueran las conclusiones a que lleguemos acerca de cómo esa hegemonía se implantó tan rápidamente, seríamos insensatos si pasáramos por alto el papel del terror, el que además de ser un estado fisiológico lo es también social, y cuyos rasgos especiales le permiten servir como el mediador por excelencia de la hegemonía colonial: el espacio de muerte donde el indio, el africano y el blanco dieron a luz un Nuevo Mundo.

En su etnografia experimental realizada en el valle del Sibundoy colombiano, en la que intenta dar cuenta de la relación entre indígenas y colonos en el proceso de extracción de caucho, Taussig afirma que la condición de posibilidad o mediador necesario para que el Estado colonial se convierta en lo que denominan Das y Poole (2004) un operador cultural universal, no es otra cosa que la construcción de una cultura del terror.

En un trabajo posterior, Taussig no restringe el análisis a la realidad que construye el Estado colonial, sino que lo extiende a la realidad que construye de manera general, el Estado moderno. En efecto Taussig dirige su mirada a las prácticas mediante las cuales acciones violentas ejercidas por el Estado son consideradas como legitimas, es decir, son investidas de racionalidad. a ese tipo de prácticas de 'racionalización de la violencia', lo llama 'fetichismo de Estado', es precisamente la acción conjunta y permanente de razón y violencia en el Estado la que crea en un mundo secular y moderno, la grandeza de la E mayúscula a la hora 
de referirnos a nuestro Estado -piénsese, en ese mismo sentido, cómo escribimos los dioses de otros con minúscula y el nuestro con mayúscula-. Al igual que todo fetichismo activo, podemos ver claramente los fetiches de otros, pero dificilmente los nuestros. En efecto, fácilmente podemos identificar la acción conjunta de la razón y la violencia en la formación de los antiguos Estados de China, Egipto, Perú o incluso del absolutismo europeo, pero no la vemos en la racionalidad legal sobre la cual se fundan y operan nuestros Estados en el presente cotidiano (Taussig, 2002).

\section{Los efectos intolerables del poder en las sociedades modernas 'más allá' del Estado}

En 1976 el pensador francés Michel Foucault publicó un influyente trabajo para el analisis del poder titulado Historia de la sexualidad I. La voluntad de saber Aunque la investigación de Foucault prescinde del método etnográfico, su teoría ha sido recogida por antropólogos que la han aplicado a sus etnografias ${ }^{9}$. Para Foucault, es fundamental realizar una crítica a la hipótesis represiva del poder en las sociedades modernas. Esta hipótesis implica considerar que: 1) el poder está centralizado en un soberano, 2) cuya función es prohibir la existencia de determinada realidad, de tal suerte que, 3) el ejercicio de la soberanía consiste en la eliminación de la realidad prohibida y por consiguiente, 4) la forma de transformación social consiste en la revolución, eliminando al soberano central, o en la resistencia, desobedeciendo las normas que prescribe el soberano y practicando la realidad que este prohibe (Foucault, 1996, p. 17).

Si bien esta hipótesis es una forma adecuada para comprender el funcionamiento del poder en la época clásica, argumenta Foucault, no lo es para el análisis del poder en las sociedades modernas, pues si la época clásica se caracteriza por la propagación de disciplinas para homogeneizar la nación en torno al soberano (poder disciplinario), la

9 Ejemplo de ello es el libro editado por Jonathan Xavier Inda titulado Anthropologies of modernity. Foucault, Governmentality and Life Politics (Inda, 2005). 
modernidad se caracteriza por la emergencia de técnicas para administrar la vida (biopoder):

Desarrollo rápido durante la edad clásica de diversas disciplinas -escuelas, colegios, cuarteles, talleres; aparición también, en el campo de las prácticas políticas y las observaciones económicas, de los problemas de natalidad, longevidad, salud pública, vivienda, migración; explosión, pues, de técnicas diversas y numerosas para obtener la sujeción de los cuerpos y el control de las poblaciones. Se inicia así la era de un "bio-poder". Las dos direcciones en las cuales se desarrolla todavía aparecían netamente separadas en el siglo XVIII. En la vertiente de la disciplina figuraban instituciones como el ejército y la escuela; reflexiones sobre la táctica, el aprendizaje, la educación, el orden de las sociedades; [...]. En la vertiente de las regulaciones de población, figura la demografia, la estimación de la relación entre recursos y habitantes, los cuadros de las riquezas y su circulación, de las vidas y su probable duración. (Foucault, 1996, pp. 169-170)

Se trata entonces para el pensador francés, de abandonar la hipótesis represiva por un análisis de los discursos/dispositivos de poder que construyen un régimen para los cuerpos, un régimen para el control de la vida. Por discursos/dispositivos de poder Foucault entiende un conjunto de enunciados con pretensión de verdad que se erigen a partir de unas prácticas e instituciones que tienen como fin gobernar, domesticar y vigilar formas de vida problemáticas para el poder, bajo su encuadramiento en categoría de anormalidad. Establece Foucault que:

Mi propósito es demostrar [...] cómo, de hecho, las condiciones políticas y económicas de existencia no son un velo o un obstáculo para el sujeto de conocimiento sino aquello a través de lo cual se forman los sujetos de conocimiento y, en consecuencia, las relaciones de verdad. Sólo [sic] puede haber ciertos tipos de sujetos de conocimiento, órdenes de verdad, dominios de saber, a partir de condiciones políticas, que son como el suelo en que se forman el sujeto, los dominios de saber y las relaciones con la verdad. (Foucault, 1992a) 
En síntesis, el análisis de los discursos/dispositivos de poder ${ }^{10}$ (lleva a establecer que: 1) el poder no está centralizado en un soberano, está difuminado en todos los lugares en donde se reproducen los dispositivos; 2) su función no es prohibir la existencia de determinada realidad, sino más bien producirla, definirla, vigilarla, controlarla; 3) es en esta capacidad de producción discursiva en donde reside el ejercicio de la soberanía y por consiguiente, 4) la forma de transformación social consiste en la identificación y desmonte de dichos dispositivos:

No pretendo en absoluto negar la importancia del aparato de Estado, pero me parece [...] , para que no encalle el proceso revolucionario, una de las primeras cosas que deben comprenderse es que el poder no está localizado en el aparato de Estado, y que nada cambiará en la sociedad si no se transforman los mecanismos de poder que funcionan fuera de los aparatos de Estado, por debajo de ellos, a su lado, de una manera mucho más minuciosa, cotidiana. Si se consiguen modificar estas relaciones o hacer intolerables los efectos de poder que en ellas se propagan, se dificultará enormemente el funcionamiento de los aparatos de Estado. (Foucault, 1992b, pp. 107-108)

\section{El orden global}

Otro objeto de observación emergente para la antropología politica fue la emergencia del ordenamiento global, que parecía haberse diseñado después de la Segunda Guerra Mundial con la consolidación de la Organización de las Naciones Unidas. Sin embargo, el sociólogo norteamericano Inmanuel Wallerstein en 1975 arguyó que dicho ordenamiento fue el efecto de la construcción de un sistema-mundo en un período de larga duración, concomitante con la emergencia del capitalismo, caracterizado por la división de trabajo entre Estados centrales encargados de la producción de manufacturas y Estados periféricos encargados del suministro de materias primas. En ese sentido un sistema-mundo:

\footnotetext{
${ }^{10}$ Margo DeMello realiza una exhaustiva revisión de la forma en que los análisis de los dispositivos de poder y los trabajos etnográficos en torno a estos han constituido un campo de "Estudios sobre el cuerpo" en el que se realiza una crítica a los efectos intolerables de dichos dispositivos como la discriminación por raza, género, clase, etc. Incluso, su trabajo particular se concentra en los efectos del poder que han llevado a la discriminación contra los animales. Ver Margo De Mello (2014).
} 
Is a social system, one that has boundaries, structures, member groups, rules of legitimization and coherence. Its life is made up of the conflicting forces which hold it together by tension, and tear it apart as each group seeks eternally to remold it to its advantage. (Wallerstein, 1975, p. 84 citado por Nash, 2002, p. 206)

Para Wallerstein uno de los principales elementos que estructura las relaciones en dicho sistema-mundo consiste en la relación de asimetría que existe entre el centro y la periferia (Gledhill, 2000, p. 5).

La puesta a prueba del análisis del sistema-mundo por la etnografía implicó un nutrido cuestionamiento. En el pionero trabajo de la antropóloga norteamericana June Nash subraya al menos dos críticas. La primera radica en la pasividad de la periferia que el modelo de Wallerstein parece presentar. En efecto, los resultados que arrojan las etnografias que se realizan en lo que Wallerstein denomina 'periferias' dan cuenta de prácticas que dificilmente podrian considerarse como periféricas y de aceptación pasiva de las reglas que establecen los centros. La segunda consiste en una acusación de etnocentrismo, pues la teoría del sistema-mundo usa descripciones de los modos sociales de producción de una manera totalizante, sin tener en cuenta de nuevo, el material etnográfico y las diversas conclusiones derivadas de la antropología económica. Para Nash el uso de herramientas etnografícas como la aproximación a los casos de estudio, la distinción emic-etic y la comparación transcultural pueden ayudar a comprender de manera más adecuada la relación de las poblaciones que comienzan a derivar su subsistencia de la integración al mercado capitalista (Nash, 2002, p. 246).

Precisamente el estudio publicado en 1993 por Ana Tsing trata de recoger dichas críticas y mediante una aproximación etnográfica a la situación de los cultivadores itinerantes en la isla de Borneo (Indonesia), describe la forma en que estos pobladores trabajan por definir y redefinir su situación en la periferia del poder del Estado. Tsing identifica que la situación periférica es un efecto de la construcción politica y cultural de una marginalidad, moldeada a partir de las normas que produce el Estado, la formación regional y étnica 
de identidades y la diferencia de género que reproducen las instituciones (Tsing, 2002, p. 325).

Hasta aquí el argumento no pareciera entrar en discusión directa con el trabajo de Wallerstein, sin embargo Tsing afirma que su trabajo etnográfico puede realizar aportes relevantes a una tensión intelectual que ha dividido a los académicos que analizan el problema del margen y la periferia: se trata de una tensión en torno a las implicaciones políticas de las nociones de diferencia cultural. Una línea de académicos, entre ellos Edward Said, consideran que la idea de diferencia cultural -y el concomitante postulado ético-jurídico de protección de dicha diferencia- profundiza la asimetría construida por el sistema-mundo. Otros académicos consideran que la protección de la diferencia cultural le permite a grupos discriminados históricamente, revertir las asimetrias que hacen posible dicha discriminación (Tsing, 2002, pp. 327-328). Desde la perspectiva de Tsing es fundamental entender que las personas que viven en espacios centrales pero construidos, percibidos y gobernados por otros como márgenes, resignifican esas construcciones de tal forma que son estas resignificaciones las que hacen posible su consolidación como sujetos (Tsing, 2002, p. 334).

En un trabajo posterior Tsing argumenta que los universales que promueve el Estado en su proceso de globalización no se llenan por sí solos de manera abstracta, se llenan de manera concreta en los encuentros entre el Estado y personas de carne y hueso, lo que justifica el uso de la etnografia como método privilegiado para documentar dichos encuentros. $\mathrm{El}$ resultado de las etnografias que representan esos encuentros demuestran que los efectos del encuentro global-local son impredecibles, pues en la cotidianidad el particular puede afectar profundamente el universal. Ello en el entendido que las culturas son continuamente co-producidas en interacciones que Tsing denomina fricciones (Tsing, 2005, p.4).

De manera cercana a los hallazgos de Tsing, Arturo Escobar y Florencia Mallon, en Latinoamérica, consideran que los grupos sociales pueden usar los discursos (en el sentido usado por Foucault) liberales para mejorar su posición en los sistemas políticos. En efecto, en su etnografia de las comunidades negras del pacífico colombiano, 
Escobar subraya la capacidad que tienen estas de construir, en un contexto de hegemonía de los discursos del desarrollo, alternativas a estos que los alteran profundamente (Escobar, 1995 citado en Gledhill 2000, p. 226). Por su parte Mallon, en su análisis de la construcción de la nación mexicana y peruana, considera que en estos casos se presentó un genuino proyecto nacional y popular basado en la etnizacion 'indianidad' que fue profundamente eficaz para contrarrestar el racismo heredado de la colonia (Mallon, 1995 citado por Gledhill, 2000, p. 201).

Finalmente, en el desarrollo de la línea argumentativa según la cual la periferia del sistema-mundo es profundamente activa y dinámica, Aihwa Ong presenta su etnografia sobre los hombres de negocios chinos en el contexto de un fuerte Estado nacional que se inserta en las lógicas del capitalismo global. El resultado que arroja dicha etnografia es que dichos hombres de negocios se mueven estratégicamente entre un cosmopolitanismo y un sistema de valores en el que se asienta la pertenencia a lo local. Ong denomina esta actividad pendular de los sujetos ciudadanía flexible. Advierte Ong:

Although contemporary Chinese merchants, bankers, and managers have burst through closed burders and freed up spaces for economic activities, they also revivid premodern forms of child, gender, and class opresión, as well as strengthened authoritarina regimes in Asia. A different kind of cosmopolitical right is at play. [...] the image of the burder-running Chinese executive with no state loyalty has become an important figure in the era of pacific Rim Capital (2002, p. 353)

\section{La condición de posibilidad de la desontologización del Estado y el surgimiento de las etnografias sobre sus prácticas cotidianas}

Pese a avanzar por los caminos de la desontologización del poder y estudiar las relaciones de los sujetos con ciertas prácticas del Estado, los trabajos en antropología politica continuaban a finales del siglo XX 
con la tendencia a desarrollar etnografias a partir de la exterioridad del Estado moderno: o bien en su margen colonial, o bien en el margen de la expansión del orden global, o bien en su 'más allá', los dispositivos del poder. Sin embargo en la década de los ochenta aparece un fenómeno migratorio desde los 'márgenes' del mundo hacia las potencias 'centrales' de Europa y Norteamérica. La respuesta de los Estados 'centrales' se caracterizó por el énfasis en discursos nacionalistas para frenar las migraciones y excluir a los migrantes de los derechos de ciudadanía.

Este es el contexto de la aparición de la sociología histórica ${ }^{11}$ que va a erigir al Estado como su objeto central de estudio, rompiendo con la tradición en ciencias sociales de considerar al Estado como un objeto no empírico, como un objeto meramente ideológico ${ }^{12}$. En efecto la sociología histórica considera fundamental aproximarse al Estado como un proceso histórico caracterizado precisamente, por la puesta en marcha de unas operaciones materiales que tienen como fin convertir los intereses particulares de un grupo social en intereses de carácter general, de tal suerte que con el transcurso del tiempo y no sin fuertes disputas, se logra una parcial homogeneización de los intereses de la población (nación). Timothy Mitchel denomina "el efecto Estado" a la efectividad de convertir los intereses particulares en intereses generales (Mitchell, 2006, pp. 184-185).

A partir de este mapa de producción de conocimiento planteado hasta aquí, quisiera sugerir que considerar al Estado como una forma de gobierno en proceso de formación, es decir, no estabilizada o parcialmente estabilizada, es la condición de posibilidad central para la apertura de un nuevo paradigma epistemológico al cual llamaria la desonotologización del Estado, apertura que derivó en el surgimiento de trabajos etnográficos cuya mirada se dirige precisamente, a las prácticas cotidianas

${ }^{11}$ Los trabajos precursores que desarrollan esta perspectiva, que de manera reiterada se citan, son: (Anderson, 1974; Abrams, 1977; Anderson, 1983; Corrigan y Sayer 1985). En Chatterjee (1993; 2011) se puede encontrar una aproximación a la forma particular en que el Estado colonial se construye a partir del caso del Estado indio, entrando en discusión con el argumento universalista de Anderson (1983).

12 Para realizar un seguimiento de la concepción epistemológica de acuerdo a la cual el Estado no puede ser estudiado por las ciencias sociales se pueden consultar unas minuciosas revisiones en Abrams (2006) y Mitchell (2006). 
del Estado mediante las cuales pretende su estabilización ${ }^{13}$. Para finalizar este mapa de revisión quisiera a continuación caracterizar tres lugares o problemas comunes en los que parecieran profundizar los estudios sobre el Estado que usan la etnografia como método para el conocimiento de sus prácticas cotidianas: la observación de los mitos y los ritos del Estado, la cuestión del fundamento de la soberanía y la crítica de la noción de margen del Estado.

\section{La observación de los mitos y los ritos del Estado}

En 1998 los antropólogos daneses Thomas Blom Hansen (profesor del departamento de antropologia de la Universidad de Stanford) y Finn Stepputat (profesor del departamento de antropología de la Universidad de Copenhague) realizaron un seminario internacional que titularon States of imagination in the postcolonial World. A este seminario invitaron a diversos académicos que, 1) venían desarrollando sus investigaciones sobre el papel del Estado moderno en los conflictos armados, las migraciones y la cultura popular; y 2) usaban un marco epistemológico común que problematizaba la idea del Estado como algo dado e inamovible, es decir, que entendían al Estado moderno como un proceso histórico de construcción (Hansen y Stepputat, 2001, p. 1).

Los participantes en el seminario coinciden en que en sus trabajos de campo siempre se encuentran con una imagen del Estado como la fuente de la soberanía a través de la cual se consolida el orden social que define un espacio para la nación y que se materializa en fronteras, infraestructura, monumentos y ciertas instituciones investidas de autoridad. A esta imagen la denominan el mito del Estado. Lo interesante aquí, arguyen los antropólogos daneses, es que este mito del Estado

${ }^{13}$ Como trabajos etnográficos pioneros que analizan la pretensión de estabilización de estas formas de gobierno y sus particularidades se puede citar para África el estudio del sociólogo francés, Jean Francois Bayart. Una de la concusiones de su estudio es la distancia que existe entre Estado y sociedad civil en los Estados poscoloniales africanos, es decir, la ausencia de representación del Estado frente a la sociedad civil. (Bayart, 1986, p. 113 citado por Gledhill, 2000, p. 101). Para Latinoamérica Deborah Poole realizó un trabajo sobre las prácticas de gobierno que el Estado peruano ha usado para poder penetrar la región de Chumbivilcas en donde juega un papel central la figura del gamonal (Poole, 1988). Finalmente, el estudio del antropólogo chileno, Claudio Lomnitz, sobre la construcción del Estado mexicano analiza la centralidad de la figura del caciquismo como forma de gobierno que aplaza la estructura burocrática abstracta, 'racional' y democrática que encarna la idea misma de Estado (Lomnitz, 1992, pp. 307-308 citado en Gledhill, 2000, pp. 113-114). 
se constata en las experiencias cotidianas de las personas que tienen un cara a cara con el Estado, incluso cuando esta experiencia se presenta en una situación de violencia permanente, en una situación de desorden ${ }^{14}$. En ese sentido, una de las preguntas que atraviesa todos los trabajos de los participantes en el seminario consiste en determinar ¿por qué continúa esta imagen de fuente de orden aún cuando el Estado está involucrado en las experiencias de violencia? Siguiendo a Abrams (1977) y a Mitchell (2006), los participantes del seminario consideran que ello se explica por la ahistoricidad con que se presenta el Estado, por el efecto Estado que producen sus prácticas, lo que van a denominar lenguajes de estatalidad (Hansen y Stepputat, 2001, p. 5).

Los participantes coinciden que metodológicamente, esta ahistoricidad se combate con el uso de la historia y la etnografia para comprender los lenguajes de estatalidad. Se trata entonces de estudiar 1) cómo el Estado intenta hacerse real y tangible a través de la producción de símbolos, textos e iconografia y la presentación (performance) de lo producido a través de prácticas localizadas y cotidianas; y 2) cómo los destinatarios de esas presentaciones las comprenden y construyen significados en la cotidianidad del encuentro entre el Estado y la sociedad.

Sin ser exhaustivos, Hansen y Stepputat proponen que la mirada de observación se dirija hacia ciertas prácticas ${ }^{15}$ que funcionan con una intensidad propia de la estructura ritual ${ }^{16}$. Asimismo la mirada debe dirigirse hacia ciertos símbolos que se inscriben en los ritos y que están en relación con los mitos de fundación del Estado ${ }^{17}$ (Hansen y Stepputat, 2001, p. 7).

${ }^{14}$ Se puede encontrar un planteamiento análogo en el trabajo seminal de Gupta para analizar los discursos de corrupción del Estado en India (Gupta, 1995).

15 De la monopolización de la violencia -realizada por la fuerza militar y la policía-, de la recolección y el control del conocimiento de la población -como los censos-, de la producción de datos de desarrollo y administración de la economía nacional.

${ }^{16}$ En esta línea de trabajo se sugiere la revisión de las investigaciones seminales sobre las burocracias estatales (Herzfeld, 1993; Gupta, 2014). Igualmente, el interesante trabajo colombiano (Buchelli, 2015).

${ }_{17}$ El discurso legal como el lenguaje propio del Estado, rituales -como la construcción de edificios, monumentos, carreteras- y la reproducción en la educación de una historia, geografia y cultura común. 


\section{El 11 de septiembre y el retorno de la cuestión del fundamento de la soberania}

Dos años después de este seminario, Hansen y Stepputat realizan un nuevo seminario para darle continuidad al diálogo sobre el Estado en el mundo postcolonial. A este seminario del año 2000 y a la publicación en 2005 del libro que recopila los trabajos de investigación, se integran académicos que han venido trabajando sistemáticamente con los problemas planteados en el primer seminario, entre los que se puede mencionar a Partha Chatterjee, Aihwa Ong, Yael Navaro-Yashin y Achille Mbembe. Este último organizó en Johannesburgo en febrero de 2003 -en el ambiente político que siguió a los hechos acaecidos el 11 de septiembre de 2001 en la ciudad de Nueva York- un seminario titulado Death, Biopolitics and Sovereignity, fundamental para entender las preocupaciones emergentes de los etnógrafos de las prácticas cotidianas del Estado (Hansen y Stepputat, 2005, p. 1).

En el ámbito del derecho internacional y las relaciones internacionales -obviamente alteradas por el ataque de Al-Qaeda al corazón de los Estados Unidos y por la respuesta de estos mediante su Guerra contra el terror- se ha identificado como fuente de soberanía de un Estado, el reconocimiento exterior que de esta hacen los otros Estados. Coinciden los trabajos compilados en 2005 por Hansen y Stepputat que este postulado no explica en ningún caso, la fuente de soberanía del Estado, más bien la supone y trata a la soberanía como un efecto sin explicar su causa. En otras palabras, este postulado naturaliza/ontologiza al Estado. Consideran entonces que el proyecto de las etnografias sobre las prácticas cotidianas del Estado es fundamental para entender la fuente de la soberanía de un Estado que puede ser hallada en el ejercicio cotidiano de la violencia sobre espacios, cuerpos y poblaciones colocados en Estados de excepción. Es esta capacidad 'exclusiva' del Estado la que en último término, otro Estado que tiene la misma capacidad, reconoce al declararlo como soberano (Hansen y Stepputat, 2005, p. 2).

El uso indiscriminado del Estado de excepción en la coyuntura post 11 de septiembre problematiza la idea según la cual el Estado 
de excepción es propio de los espacios de frontera/colonización, o de momentos límite al interior del Estado de normalidad. Para abordar esta cuestión las etnografias van a ser leídas a través de teorías que se apartan de las nociones liberales de soberanía destinadas a sostener el mito del Estado (soberanía nacional-soberanía popular) como forma 'racional' de administración del poder en las sociedades modernas. Los antropólogos se van a familiarizar con el debate sobre el fundamento de la soberanía que se produjo en Alemania con ocasión del ascenso del nacionalsocialismo al poder, debate trenzado entre el abogado católico Carl Schmitt y el pensador judío Walter Benjamin.

Ambos coincidian en que la transición política de la modernidad caracterizada por la sustitución de un fundamento teológico de la soberanía por un fundamento secular era la raíz de una alteración profunda del ordenamiento social, propiamente hablando, era la raíz de una crisis social. Para Schmitt es en la crisis que se puede distinguir al soberano, no por el cumplimiento de los procedimientos liberales en el marco de un Estado de derecho preestablecido, sino más bien por ser aquel que pueda, en términos reales de fuerza, decidir el Estado de excepción (ausnhame). Es decir que, aquel que pueda en determinado tiempo y lugar, funda el ordenamiento y lo hace valer en la sociedad (Schmitt, 2009).

Para Benjamin esta noción sin fundamento democrático de la soberanía es la base del gobierno del 'fascismo' y es una fórmula promotora de la violencia en la sociedad. Por ello, sin negar la permanente inestabilidad, ambigüedad e indefinición del fundamento de la soberanía en la sociedad moderna (como quisieran los liberales), considera que precisamente por dicha ausencia de fundamento, la soberania debe ser escrutada y criticada cotidianamente para identificar un 'momento de peligro' en el que esta es ejercida para desaparecer la humanidad de los sujetos (Benjamin, 2007-2015, p. 308).

La introducción de la cuestión del fundamento de la soberanía, de la mano del debate Schmitt-Benjamin y desarrollado por otros autores como Bataille, Foucault, Agamben, Zizek, entre otros, hizo que las etnografias sobre los Estados poscoloniales pasaran ahora hacia 
los Estados del 'centro', dejando de un lado el acento en la 'diferencia colonial' y produciendo un interesante debate sobre la cuestión del significado del 'margen' del Estado ${ }^{18}$.

\section{La aproximación critica a la noción de margen del Estado}

De manera simultánea al seminario organizado por Mbembe en el 2003, las profesoras del departamento de antropología de Johns Hopkins University, Veena Das y Deborah Poole, realizaron un seminario en Santa Fe, New Mexico (School of American Research) en el cual se preguntaban por la forma en que el Estado podía ser repensado como un objeto etnográfico. Después de revisar la aproximación de Hansen y Stepputat que acentúa las etnografias en el Estado poscolonial (la diferencia colonial), Das y Poole invitan a tomarse en serio la crítica a la noción liberal de soberanía citada arriba y en ese sentido, a crear una antropología del Estado a partir de etnografias que describan las prácticas cotidianas en los márgenes que el Estado mismo construye (Das y Poole, 2004, pp. 5-6). Para ello es necesario un replanteamiento crítico de la noción de margen, lo que implica comprender la violencia del Estado en contra de ciertos grupos sociales, no desde una perspectiva en que interpreta la violencia como lo excepcional del Estado moderno, sino como su condición de posibilidad (Das y Poole, 2004, pp. 14-15).

Las etnografias de Estado que recopilan Das y Poole permiten realizar una aproximación crítica a la noción de margen, al menos en dos sentidos. En primer lugar se advierte que el Estado construye márgenes en relación a tres ámbitos diferentes: el ámbito espacial, en el que requiere diferenciar constantemente centro/periferia y construir fronteras visibles / invisibles ${ }^{19}$; en el ámbito de la legibilidad en el que precisa distinguir los sujetos que pueden leer y contar, y pueden

\footnotetext{
${ }^{18}$ Una interesante variación de este problema se puede seguir en los recientes trabajos etnográficos sobre prácticas cotidianas del Estado que observan los periodos de transición política o lo que se llaman procesos de justicia transicional. Al respecto ver Hinton (2011), Castillejo (2009; 2015), Guglielmucci (2013) y Jaramilo (2014).

19 Sobre la importancia de la noción de frontera como expresión primordial del Estado se puede revisar el trabajo de Yael Navaro-Yashin (2005, p. 111).
} 
ser leídos y contados; y en el ámbito de las formas de vida, donde se pretende clasificar las conductas humanas en normales y patológicas (Das y Poole, 2004, pp. 8-9).

En segundo lugar es preciso subrayar que los márgenes no son inertes: los espacios de excepción que se constituyen en los márgenes son espacios abiertos a la creatividad, a la discusión sobre alternativas de las formas económicas y políticas instituidas. Así, los márgenes pueden ser espacios propicios para lo profundización de asimetrías o para el establecimiento de nuevos equilibrios. (Das y Poole, 2004, p. 19) ${ }^{20}$.

En sintesis, los hallazgos de las etnografias sobre las prácticas cotidianas del Estado moderno nos dejan una imagen muy distinta de aquella que promueve la mitología liberal y que lo ubica como el garante universal de los derechos del ciudadano. Es en ese sentido que producen una desontologización. A través de esta, dichas etnografias nos permiten hacer evidente el despliegue de múltiples mitologías y rituales, en nombre de esa forma de gobierno que llamamos 'estatalidad' y que tienen como fin estabilizar el fundamento del ejercicio de la soberanía, en un contexto en el que esta soberanía se encuentra siempre en disputa, por la ausencia misma de un único fundamento. La disputa por estabilizar el fundamento que nos muestran las etnografias de las prácticas cotidianas hace de eso que llamamos Estado un aparato que, de manera permanente, es usado para diseñar márgenes sobre poblaciones y territorios con el fin de ubicarlos en la centralidad o en la marginalidad de los planes de gobierno que se implementan desde dicho aparato.

${ }^{20} \mathrm{Al}$ respecto, es fundamental observar cómo las personas o comunidades se movilizan de forma estratégica y creativa por dentro y por fuera de las márgenes con el objeto de reivindicar derechos o resistir a prácticas arbitrarias. Un ejemplo son las Comunidades de paz en Colombia como forma de contestar el desplazamiento desde los márgenes (Sanford, 2004). 


\section{Referencias}

Abrams, P. (1977). Notes on the Difficulty of Studying the State. British Sociological Association. Annual Conference Paper.

Abrams, P. (2006). Notes on the Difficulty of Studying the State. En A. Sharma, y A. Gupta (Eds.), The Anthropology of the State. A reader (pp.58-89). Oxford: Blackwell Publishing.

Alexandrowicz, C. H. (1971). The juridical expression of the sacred trust of civilization. The American Journal of International Law, 65(1), 149-159.

Anderson, P. (1974). Lineages of the Absolutist State. London: New Left Books.

Anderson, B. (1983). Imagined Communities. Reflections on the Origins and Spread of Nationalism. London: Verso.

Anderson, B. (1993). Comunidades imaginadas: refelxiones sobre el origen y difusión del nacionalismo. México: Fondo de Cultura Económica.

Asad, T. (2002). From the History of Colonial Anthropology to the Anthropology of Western Hegemony. En J. Vincent. The Anthropology of Politics. A Reader in Etnography, Theory and Critique (pp. 133-141). Oxford: Blackwell Publishing.

Benjamin, W. (2007-2015). Sobre el concepto de la historia. En W. Benjamin, Obras (Vol. II, p. 308). Madrid: Abada Editores.

Bourdieu, P. (1993). Génesis y estructura del campo burocrático. Actes de la Recherche en Scineces Sociales, (96-97), 49-62.

Bourdieu, P. (1997). Espíritus de Estado. Génesis y Estructura del Campo Burocrático. En P. Bourdieu, Razones prácticas. Sobre la teoría de la acción. (pp. 91-125). Barcelona: Editorial Anagrama.

Buchelli, L. (2015). Activismo burocrático. La construcción cotidiana del principio de legalidad. Bogotá: Ediciones Uniandes. 
Cárdenas-Támara, F. (2014). Reconstitución de la antropología política. Heternonomías entre la ciencia política y la antropología. Análisis Político, (80), 211-226.

Castillejo, A. (2009). Archivos del dolor: ensayos sobre la violencia y el recuerdo colectivo en la Sudáfrica contemporánea. Bogotá: Universidad de los Andes.

Castillejo, A. (2015). La imaginación social del porvenir: reflexiones sobre Colombia y el prospecto de una Comisión de la Verdad. Buenos Aires: Clacso.

Chatterjee, P. (1993). The Nation and its Fragments. Princeton, New Jersey: Princeton University Press.

Chatterjee, P. (2008). La nación en tiempo heterogéneo. Buenos Aires: Siglo XXI Editores.

Chatterjee, P. (2011). Lineages of political society. New York: Colombia University Press.

Clastres, P. (2001). Investigaciones en Antropología Politica. Barcelona: Gedisa Editorial .

Corrigan, P. y Sayer, D. (1985). The Great Arch: English State Formation as Cultural Revolution. Oxford: Basil Blackwell.

Das, V. y Poole, D. (2004). Anthropology in the Margins of the State. Santa Fe: School of American Research Press.

De Mello, M. (2014). Body Studies: an introduction. New York: Routledge.

Escobar, A. (1995). Encountering Development: The Making and Unmaking of the Third World. Princeton NJ: Princeton University Press.

Evans-Pritchard, E. (2002). Nuer Politics: Structure and System. En J. Vincent, The anthropology of politics. A reader in ethnography, theory and critique (pp.35-38). Oxford: Blackwell Publishing. 
Ferguson, J. y Gupta, A. (2005). Spatializing States: Toward an Ethnography of Neoliberal Governmentality. En J. X. Inda, Anthropologies of Modernity. Foucault, Governmentality and Life Politics (pp. 105-134). Oxford: Blackwell Publishing.

Foucault, M. (1992a). La Verdad y las Formas Jurídicas. Barcelona: Gedisa Editorial.

Foucault, M. (1992b). Microfisica del poder (Tercera Edición ed.). Madrid: Las Ediciones de la Piqueta.

Foucault, M. (1996). Historia de la Sexualidad I. La Voluntad de Saber (Vigésimoquinta Edición ed.). México: Siglo Veintiuno Editores.

Gal, S. (2002). Between Speech and Silence. En J. Vincent, The Anthropology of Politics. A reader in Ethnography, Theory and Critique (pp. 213-221). Oxford: Blackwell Publishing.

Gledhill, J. (2000). Power and its Disguises. Anthropological Perspectives on Politics (2 ${ }^{\mathrm{a}}$ Edición ed.). London: Pluto Press.

Gough, K. (2002). New Proposals for Anthropologists. En J. Vincent, The Anthropology of politics. A reader in Ethnography, Theory and Critique (pp. 110119). Oxford: Blackwell Publishing.

Guglielmucci, A. (2013). La consagración de la memoria: una etnografía acerca de la institucionalización del recurso sobre los crimenes del terrorismo del Estado en la Argentina. Buenos Aires: Antropofagia.

Gupta, A. (1995). Blurred Boundaries: The Discourse of Corruption, the Culture of Politics, and the Imagined State. America Ethnologist 22(2), 375-402.

Gupta, A. (2014). Las burocracias. Una aproximación distinta en que pensamos el Estado. Bogotá: Siglo del Hombre Editores.

Hansen, T. B. y Stepputat, F. (2001). States of imagination. Ethnographic Explorations of the Postcolonial State. Durham: Duke University Press. 
Hansen, T. B. y Stepputat, F. (2005). Sovereign Bodies. Citizens, Migrants, and States in the Postcolonial World . Princeton, New Jersey: Princeton University Press.

Hart, H. (1998). El concepto de derecho. Buenos Aires: Abeledo Perrot.

Herzfeld, M. (1993). The social production of indifference. Exploring the symbolic roots of western bureaucracies. Chicago: The Chicago University Press.

Hinton, A. L. (2011). Introduction: toward an anthropology of transitional justice. En A. L. Hinton, Transitional Justice: Global mechanisms and local realities after genocide and mass violence (pp. 1-25). New Jersey: Rutgers University Press.

Inda, J. X. (2005). Anthropologies of Modernity. Foucault, Governamentality and Life Politics. Oxford: Blackwell Publishing.

Jaramilo, P. (2014). Etnicidad y victimización: genealogías de la violencia y la indigenidad en el norte de Colombia. Bogotá: Ediciones Uniandes.

Kurtz, D. V. (2001). Political Anthropology. Paradigms and Power. Boulder, Colorado: Westview Press.

Laban, A. (2011). Introdution: toward an anthropology of transitional justice. En A. Laban, Transitional Justice: Global mechanisms and local realities after genocide and mass violence (pp. 1-25). New Jersey: Rutgers University Press.

Lewellen, T. C. (2003). Political Anthropology. An Introduction. Westport: Praeger.

Lomnitz, C. (1992). Exits from the Labyrinth:Culture and Ideology in the Mexcan National Espace. Berkeley: University of California Press.

Mallon, F. E. (1995). Peasant and Nation: The making of Postcolonial Mexico and Peru. Berkeley: University of California Press.

Mamdani, M. (1996). Citizen and Subject. Contemporary Africa and the Legacy of Late Colonialism. Princeton, New Jersey: Princeton University Press. 
Mamdani, M. (2012). Define and rule: native as political identity. Cambridge, Massachussetts: Harvard University Press.

Marcus, G. y Fischer, M. (1986). La antropología como crítica cultural. Un momento experimental en las ciencias humanas. Buenos Aires: Amorrortu Editores.

Mitchell, T. (2006). Society, Economy, and the State Effect. En A. Sharma y A. Gupta (Eds.). The Anhtropology of the State. A reader (pp.76-97). Oxford: Blackwell Publishing.

Nash, J. (2002). Ethnographic Aspects of the World of Capitalist System. En J. Vincent. The Anthropology of Politics. A reader in Ethnography, Theory and Critique (pp. 234-247). Oxford: Blackwell Publishing.

Navaro-Yashin, Y. (2005). Confinement and the Imagination: Sovereignty and Subjectivity in a Quasi-State. En T. B. Hansen y F. Stepputat, Sovereign Bodies. Citizens, Migrants, and States in the Postcolonial World (pp. 103-119). Princeton, NJ: Princeton University Press.

Ong, A. (2002). Flexible Citizenship among Chinese Cosmopolitans. En J. Vincent. The Anthropology of Politics (pp. 338-355). Oxford: Blackwell Publishing.

Ortner, S. (1995). Resistance and the problem of ethnographical refusal. Comparative Studies in Society and History 37(1), 173-193.

Poole, D. (1988). Landscapes of power in a Cattle-Rsutling culture of southern andean Peru. Dailectical Anthropology, 12, 367-398.

Said, E. (1979). Orientalism. New York: Vintage Books Editions.

Sanford, V. (2004). Contesting displacement in Colombia: Citizenship and State Sovereinity at the Margins. En V. Das y D. Poole (Eds.). Anthropology in the margins of the state (pp. 253 -278). Santa Fe: School of American Research Press.

Schmitt, C. (2009). Teología politica. Madrid: Editorial Trotta. 
Scott, J. C. (1977). The Moral Economy of the Peasant. Rebellion and Subsitence in Southeast Asia. New Haven: Yale University Press.

Scott, J. C. (1985). Weapons of the weak. Everyday Forms of Peasant Resistance. New Haven: Yale University Press.

Swartz, M. J., Turner, V. W. y Tuden, A. (2002). Political Anthropology. En J. Vincent,. The Anthropolgy of Politics. A reader in ethnography, theory and critique (pp. 102-109). London: Blackwell publishing.

Taussig, M. (2002). Chamanismo, colonialismo y el hombre salvaje. Un estudio sobre el terror y la curación. Bogotá: Grupo Editorial Norma.

Tsing, A. (2002). Politics on the periphery. En J. Vincent, The anthropology of politics. A reader in Ethnography, Theories and Critique (pp. 325-337). Oxford: Blackwell Publishing.

Tsing, A. (2005). Friction. Ethnography of global connection. New Jersey: Princeton University Press.

Vincent, J. (2002). The Anthropology of Politics. A Reader in Ethnography, Theory and Critique. Oxford: Blackwell Publishing.

Wallerstein, I. (1975). The Modern World System: Capitalist Agriculture and the Origins of the European World Economy in the Sixteenth Century. New York : Academic.

Weber, M. (2006). Bureaucreacy. En A. Sharma y A. Gupta, The anthropology of the state. A reader (pp. 49-70). Oxford: Blackwell Publishing.

\section{Cómo citar este artículo}

García, J. F. (2016). Los aportes epistemológicos del pensamiento antropológico sobre el Estado moderno y las etnografias sobre sus prácticas cotidianas. Universitas Humanística, 82, 105-134. http: / dx.doi.org/ 10.11144/Javeriana. uh82.aepa 